\title{
Comparison of CA Model for Dendritic Solidification with Scheil and Equilibrium Models
}

\begin{abstract}
A. ZYSKA*, K. Boroń, And S. DOMin
Częstochowa University of Technology, Faculty of Production Engineering and Materials Technology, Institute of Metallurgy and Metal Technology, al. Armii Krajowej 19, 42-200 Częstochowa, Poland

(Received August 22, 2019; revised version February 6, 2020; in final form February 11, 2020)

A mathematical model based on the technique of a cellular automaton to simulate the dendritic solidification of two-component alloys is presented. The results of numerical calculations were compared with the predictions of the Scheil and equilibrium analytic models. Comparative analysis was carried out on the example of Al- $3 \mathrm{wt} \% \mathrm{Mg}$ alloy. The differences between the models were revealed by determining the profiles of the solid phase Mg concentration for the three cooling rates 1,25 , and $70 \mathrm{~K} / \mathrm{s}$, assuming no diffusion and limited diffusivity in the solid state. It has been found that the degree of microsegregation of the element in the test alloy is mainly controlled by the cooling rate, and the effects of back diffusion are detectable only at low intensity of heat dissipation. Moreover, the developed $2 \mathrm{D}$ cellular automaton model allows simulation of the evolution of dendritic structures and determination of solid compositions' variations for different solidification conditions in the range from predictions of the equilibrium model to the predictions of the Scheil model.
\end{abstract}

DOI: 10.12693/APhysPolA.137.1046

PACS/topics: CA model, solidification, Al-Mg alloy, microsegregation

\section{Introduction}

Currently, modelling of the dendritic structure in castings is performed using the cellular automaton method (CA) or the phase field method (PF) [1-5]. In these models, the equations of mass and heat transport as well as relations describing the solidification front, its energy, curvature, and surface tension anisotropy are solved in a coupled manner. The mathematical apparatus of models allows to capture the real solidification conditions, taking into account the local microsegregation of the alloy component, the interaction of solute fields, and the local interaction between growing dendrites. Numerical simulations reproduce very realistically the formation of dendrite main arms and their growth, nucleation, and development of the second or higher order arms and their eventual fusion in the final solidification stage [3-8]. A fundamental quantity which describes the movement of the solidification front is the growth velocity. In the main group of CA models, it is determined based on solute balance or solute flux balance at the solid/liquid interface $[3-6,8]$. The concentration gradient occurring on the transformation front affects the diffusion rate, the morphology of the structures, the solute distribution in the liquid and solid phase during solidification, and, as a result, the final microsegregation in the solidified casting.

On a macroscopic scale, solidification of metal alloys is most often considered for equilibrium conditions or based on the Scheil equation assuming no diffusion in solid [9]. The Scheil model is used for alloys containing

*corresponding author; e-mail: zyska@wip.pcz.pl components with low values of solid phase diffusion coefficient, solidifying in a wide range of temperatures, while the equilibrium solidification model is applied to alloys containing components with high values of this coefficient or alloys that solidify at very low heat dissipation rate. However, in the dominant group of castings produced by basic casting techniques, solidification has an indirect characteristic. Then the average concentration of the component and its distribution in solid during solidification is between the values resulting from the equilibrium model and the Scheil model. The aim of this work is to compare the simulation results of the developed CA model with analytical predictions of Scheil and equilibrium models, which are often applied to model numerical solidification and experimental research $[10,11]$.

\section{Governing equations}

The CA was determined for a planar area divided into uniform square cells with side length $\Delta x$. The state of each cell was described by the following basic quantities: fraction of the solid phase $g_{S}$, temperature $T$, concentration of solute $C$, phase state $F$, preferential crystallographic orientation angle $\theta_{0}$, the average curvature of the interface $\kappa$, and the direction normal to the interface $\varphi$. Two types of neighbourhoods were used in the calculation procedure. The von Neumann neighbourhood was adopted for the energy and mass transport phenomena, while the Moore neighbourhood was applied for all other cases. The calculations were started by randomly determining the position of the nucleation cells and assigning them also a randomly preferential crystallographic orientation $[2,6,12]$. Scanning the automaton was carried out on blocks of the elementary cell with the dimensions $3 \times 3$. 
During solidification, cells change their state from liquid $\left(g_{S}=0\right)$ to interface $\left(0<g_{S}<1\right)$, and then to solid $\left(g_{S}=1\right)$. At the moment when the solid fraction in a cell reaches 1 , its state is changed to solid, and the neighbouring cells in liquid are captured to the interface, inheriting the preferential crystallographic orientation. The basis of the applied cellular automaton consists of the three fields: the temperature field, the solute concentration field, and the solid phase fraction field. They reproduce the kinetics of the dendritic structure growth both in quantitative and qualitative aspects. The local position of the solidification front and the local shape of dendrites are obtained by the coupled solution of model equation system. The mathematical form of the developed CA model in a twodimensional computational domain is expressed by

- energy equation

$$
\rho c_{p} \frac{\partial T}{\partial t}=\frac{\partial}{\partial x}\left(\lambda \frac{\partial T}{\partial x}\right)+\frac{\partial}{\partial y}\left(\lambda \frac{\partial T}{\partial y}\right)-\rho L \frac{\partial g_{S}}{\partial t},
$$

where $g_{S}$ is solid fraction, $t$ is time, $L$ is latent heat, $\rho$ is density, $T$ is temperature, $\lambda$ is thermal conductivity, and $c_{p}$ is specific heat.

- solute conservation equation

$$
\frac{\partial C_{R}}{\partial t}=D_{R}\left(\frac{\partial^{2} C_{R}}{\partial x^{2}}+\frac{\partial^{2} C_{R}}{\partial y^{2}}\right)+C_{R}(1-k) \frac{\partial g_{S}}{\partial t},
$$

where $C_{R}$ is solute concentration determined by $C_{L}$ for liquid and $C_{S}$ for solid with appropriate $D_{L}$ and $D_{S}$ as solute diffusion coefficients, and $k$ is partition coefficient.

- equilibrium temperature at the solid/liquid interface

$$
T^{F}=T_{L}+\left(C_{L}^{F}-C_{0}\right) m_{L}-\Gamma \bar{\kappa} f\left(\phi, \theta_{0}\right),
$$

where $\Gamma$ is the Gibbs-Thomson coefficient, $\kappa$ is interface curvature, $f\left(\phi, \theta_{0}\right)$ is anisotropy function, $\phi$ is growth angle, $\theta_{0}$ is preferential crystallographic orientation angle, $C_{L}^{F}$ is solute concentration at the interface, $C_{0}$ is solute concentration in the alloy, $m_{L}$ is slope of the liquid, and $T_{L}$ is equilibrium liquid temperature.

- equation on the average curvature of the interface [13]:

$$
\bar{\kappa}=-(\nabla \cdot \hat{n})=\frac{1}{|n|}\left[\left(\frac{n}{|n|} \cdot \nabla\right)|n|-(\nabla \cdot n)\right],
$$

where $\boldsymbol{n}$ is normal unit vector to the solidification front. - anisotropy function

$$
f\left(\phi, \theta_{0}\right)=\left[1-\delta \cos \left(m_{S}\left(\phi-\theta_{0}\right)\right)\right],
$$

where $m_{s}$ is crystal-order parameter, for cubic symmetry $m_{s}=4, \delta$ is the anisotropy coefficient.

- growth angle between the normal direction to the interface and the reference direction $0 x$

$$
\phi=\arctan \left(\frac{M_{x}}{M_{y}}\right),
$$

where $M_{x}$ and $M_{y}$ indicate moments of the system relative to the coordinates $0 x$ and $0 y$, respectively. They are defined as:

$$
\begin{aligned}
& M_{x}=\sum_{i=1}^{6} g_{S, i}^{y} \Delta x, \\
& M_{y}=\sum_{i=1}^{6} g_{S, i}^{x} \Delta y,
\end{aligned}
$$

where $g_{S, i}^{x}$ is solid fractions in cells $g^{N E}, g^{E}, g^{S E}$, $g^{N W}, g^{W}, g^{S W}, g_{S, i}^{y}$ are solid fractions in cells $g^{N W}, g^{N}$, $g^{N E}, g^{S W}, g^{S}, g^{S E}$, the superscripts being related to the position of cells named according to the compass rose.

At each time step interval $\Delta t$, the solid phase increase in interface cells is calculated by comparing the local interface equilibrium concentration $C_{L}^{F}$ determined by the transformed formula (3) with the actual liquid concentration $C_{L}$ from (2). If, after the comparison the difference is greater than zero $\Delta C=C_{L}^{F}-C_{L}>0$, then in the interface cell the solid fraction increases according to the relation

$$
\Delta g_{S}=\left(C_{L}^{F}-C_{L}\right) /\left(C_{L}^{F}-C_{S}^{F}\right) .
$$

\section{Simulation results and discussion}

Numerical simulations of dendritic solidification were performed for the alloy $\mathrm{Al}-3 \mathrm{wt} \% \mathrm{Mg}$ on a cellular automaton with a size of $256 \times 256$ cells. The dimension of the unit cell was $2 \mu \mathrm{m}$. The periodic boundary condition for the diffusion equation and the Neumann boundary condition for the heat transport equation were assumed for the domain walls. At the initial time, the temperature and concentration distributions in the whole area were homogeneous and were defined as $T_{0}$ and $C_{0}$, respectively. After reaching the liquid temperature, 8 nuclei were randomly placed in the domain, assigning them

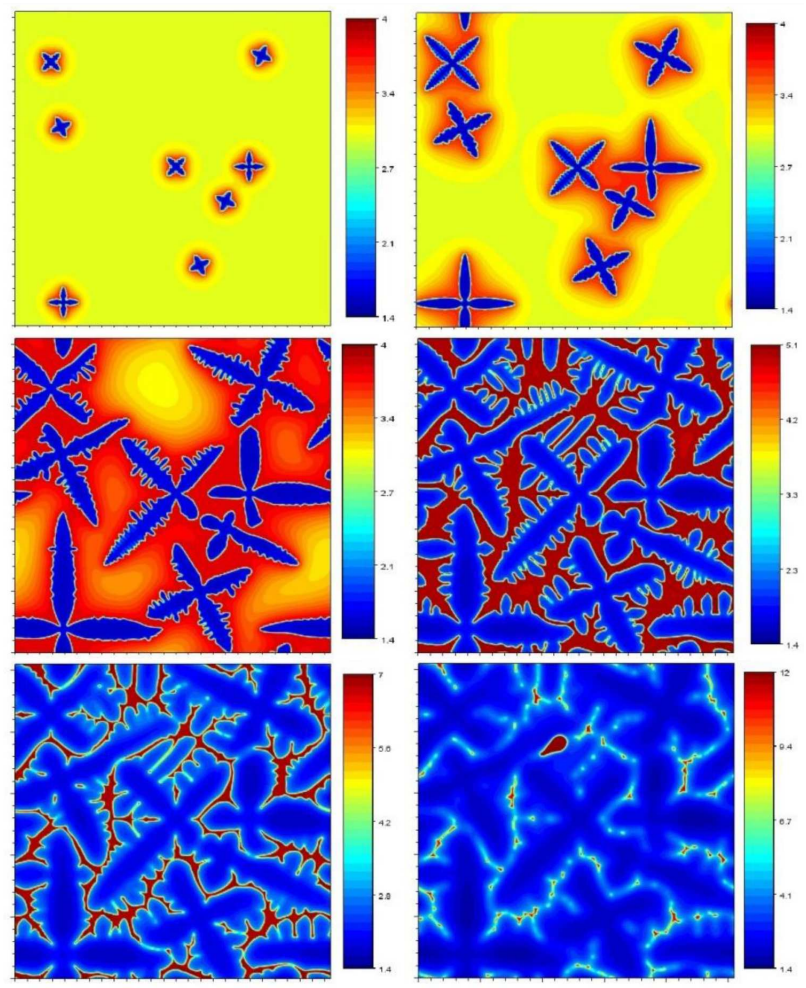

Fig. 1. Evolution of dendrites in the $\mathrm{Al}-3 \mathrm{wt} \% \mathrm{Mg}$ alloy at a cooling rate of $25 \mathrm{~K} / \mathrm{s}$ with solute distribution fields. 


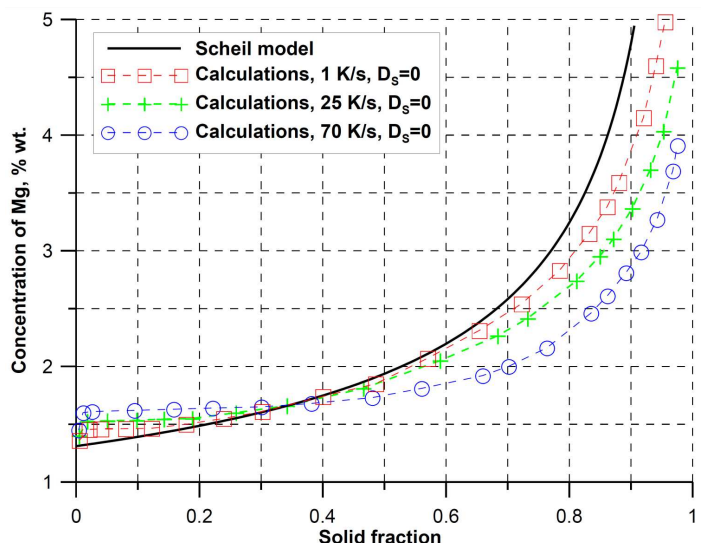

Fig. 2. Solid phase $\mathrm{Mg}$ concentration profiles obtained at the cooling rates $1,25,70 \mathrm{~K} / \mathrm{s}$ and the Scheil model.

a preferential crystallographic orientation angle also using randomization procedures. The evolution of dendrites and the corresponding $\mathrm{Mg}$ concentration fields in the subsequent calculation steps are shown in Fig. 1.

Comparison of the developed CA model with the Scheil and equilibrium models was carried out on the basis of solid phase $\mathrm{Mg}$ concentration profiles. In order to determine the differences between the predictions of the numerical and analytical model, two series of simulations were performed. In the first series, the effect of cooling rate on the variation in solid composition was analyzed, assuming no diffusion in the solid and limited diffusion in the liquid. The calculations were carried out for three cooling rates of $1,25,70 \mathrm{~K} / \mathrm{s}$, and the Scheil model, decreasing the temperature from the liquidus temperature to the eutectic temperature. Changes in Mg concentration in the solid phase as a function of the solid fraction are presented in Fig. 2.

Figure 2 shows that in the initial solidification stage, the solid phase $\mathrm{Mg}$ concentration for the CA model is higher than that in the Scheil model, while in the final transient period the relation is reversed. Such differences in $\mathrm{Mg}$ concentration profiles result from the assumption of the Scheil model, i.e., unlimited diffusion of the component in the liquid. Whereas, in the numerical model the concentration gradient occurring on the solidification front and in the liquid is taken into account. Based on the characteristics determined from the CA model, it can be concluded that the inhomogeneity of liquid and differences in the results of both models increase as the cooling rate increases. Simulations also showed that the increment of solid fraction (dendrites) in the initial and middle stages of solidification proceeds at very small variations in $\mathrm{Mg}$ concentration. Such solid phase increment corresponds to solidification under steady state conditions. The papers $[6,12,14]$ compared CA solidification models with the LGK [15] analytical model of steady state dendrite tip growth. It was found that for small and medium undercooling (cooling rates) in binary and multi-component alloy melts there is a high consistency

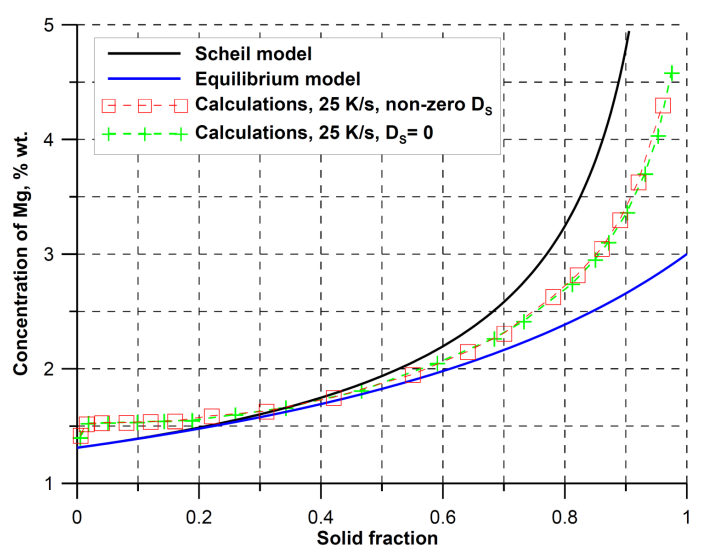

Fig. 3. Comparison between analytical models and the CA model for prediction of solid composition assuming no and limited diffusion in the solid phase and cooling rate of $25 \mathrm{~K} / \mathrm{s}$.

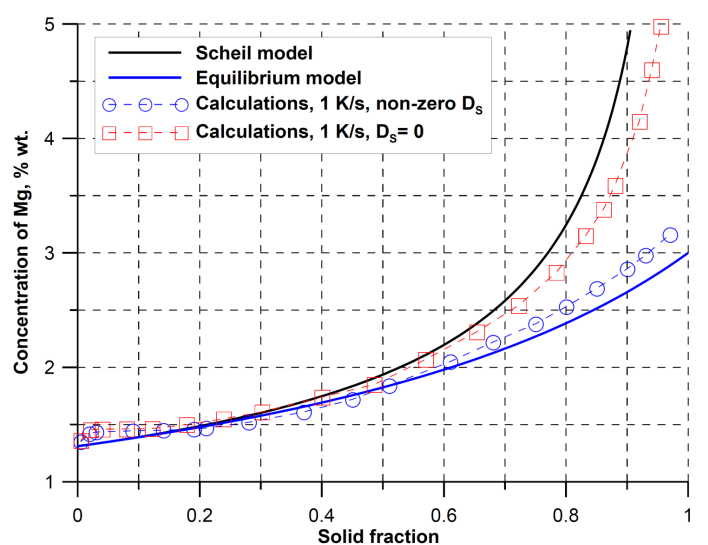

Fig. 4. Comparison between analytical models and the CA model for prediction of solid composition assuming no and limited diffusion in the solid phase and cooling rate of $1 \mathrm{~K} / \mathrm{s}$.

of concentration profiles obtained in both approaches. In addition, the study [12] showed that the modeled solute distribution during solidification of SCN-Ace alloy system corresponds to the experimental data.

The second series of simulations concerned the investigation of the effect of solid phase diffusion on the course of the solidification process and microsegregation. The calculations were made for two cooling rates of $1 \mathrm{~K} / \mathrm{s}$ and $25 \mathrm{~K} / \mathrm{s}$, assuming limited diffusion in the liquid and solid. The comparison of the results from the numerical model with the predictions of analytical models is presented in Figs. 3 and 4.

At high cooling rates (Fig. 3), solid phase diffusion processes have no significant effect on the solidification behaviour of the alloy. The curves obtained for the diffusion coefficient set to zero and non-zero almost coincide with each other. For a cooling rate of $25 \mathrm{~K} / \mathrm{s}$, the total solidification time is too short for back diffusion to be detected. 


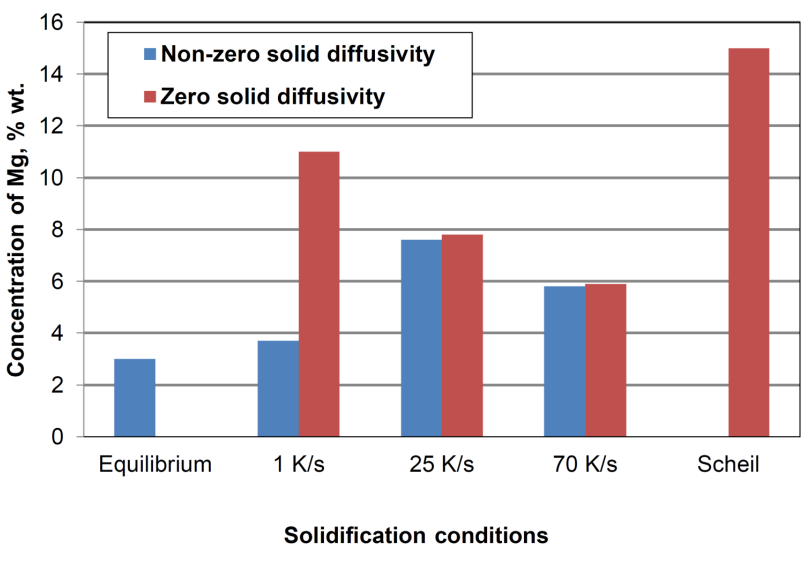

Fig. 5. Mg concentration at the solidification front for different cooling rates and diffusion coefficient values the solid fraction equal to 0.985 .

The phenomenon of solid phase diffusion is evident in the case of solidification with low intensity of heat dissipation (Fig. 4). In this variant, back diffusion leads to the homogenization of the solute in the dendrites, which results in a significant shift of the $\mathrm{Mg}$ concentration profile towards the equilibrium model predictions. The solid composition variation during growth is quite different, assuming no diffusion $D_{S}=0$. The determined curve is the closest to the one calculated from the Scheil model. Zhu and Stefanescu [12] presented similar characteristics and solute concentration profiles by comparing the Scheil model with their own virtal CA model on the example of $\mathrm{Al}-\mathrm{Cu}$ binary alloy solidification.

Figure 5 shows the concentration of $\mathrm{Mg}$ at the solidification front for different cooling rates and diffusion coefficient values. The calculations were made for a solid fraction equal to 0.985 , which corresponds to the achievement of the eutectic point composition in the Scheil model. The highest solute microsegregation arises for the solidification according to the Scheil model and the CA model with the assumptions of low cooling rate and zero diffusion coefficient. With the increase of the cooling rate, the growth rate of dendrites as well as the concentration of $\mathrm{Mg}$ increase in the interface cells in the initial and middle stages of solidification. As a result, the solid solidifying from magnesium enriched liquid is more homogeneous despite the absence of diffusion [9]. The lowest microsegregation of the component and the composition of the solid phase close to the predictions of the equilibrium model are obtained for non-zero diffusion coefficient and low intensity of heat dissipation. The solid phase diffusion process at low solidification rate homogenizes the dendritic structure of the alloy. Magnesium microsegregation during solidification of nominally single-phase $\mathrm{Al}-\mathrm{Mg}$ alloys is a harmful phenomenon because it contributes to the formation of the $\mathrm{Al}_{8} \mathrm{Mg}_{5}$ phase and intercrystalline corrosion of castings [16].

\section{Summary}

The developed two-dimensional CA model allows simulation of solid phase growth for different solidification conditions. Depending on the cooling rate and the diffusion rate, it is possible to determine solidification curves that vary within a wide range from the Scheil model predictions to the equilibrium model predictions. The degree of microsegregation of an element in the tested $\mathrm{Al}-3 \mathrm{wt} \% \mathrm{Mg}$ alloy is mainly controlled by the intensity of heat dissipation. The solid phase diffusion effects are only detectable at low cooling rates. Apart from determining the solidification characteristics and local segregation, the developed model allows for realistic reproduction of dendritic structures. Using the CA model, the following phenomena are simulated: formation and coarsening of the first-order arms and initiation, evolution and fusion of the secondary arms, including the mutual interaction between the individual dendrites as they grow.

\section{References}

[1] M.A. Zaeem, H. Yin, S.D. Felicelli, Appl. Math. Modell. 37, 3495 (2013).

[2] A. Choudhury, K. Reuther, E. Wesner, M. Rettenmayr, Computat. Mater. Sci. 55, 263 (2012).

[3] A. Zyska, K. Boroń, P. Kordas, Archiv. Foundry Eng. 18, 87 (2018).

[4] K. Reuther, M. Rettenmayr, Rev. Computat. Mater. Sci. 95, 213 (2014).

[5] T. Dobravec, B. Mavrič, B. Šarler, J. Computat. Phys. 349, 351 (2017).

[6] M.F. Zhu, W. Cao, S.L. Chen, C.P. Hong, Y.A. Chang, J. Phase Equilibr. Diff. 28, 130 (2007).

[7] S. Luo, W. Wang, M. Zhu, Int. J. Heat Mass Transf. 116, 940 (2018).

[8] A. Zyska, Z. Konopka, M. Łągiewka, M. Nadolski, Archiv. Foundry Eng. 16, 99 (2016).

[9] E. Fraś, Crystallization of Metals, WNT, Warszawa 2003.

[10] D.M. Stefanescu, Science and Engineering of Casting Solidification, Springer, 2009.

[11] W. Wołczyński, A.A. Ivanova, P. Kwapisiński, E. Olejnik, Archiv. Metall. Mater. 62, 2461 (2017).

[12] M.F. Zhu, D.M. Stefanescu, Acta Mater. 55, 1741 (2007).

[13] D.B. Kothe, R.C. Mjolsness, M.D. Torrey, A Computer Program for Incompressible Flows with Free Surface, Los Alamos National Lab., Los Alamos 1991.

[14] S.C. Michelic, J.M. Thuswaldner, C. Bernhard, Acta Mater. 58, 2738 (2010).

[15] J. Lipton, M.E. Glicksman, W. Kurz, Mater. Sci. Eng. 65, 57 (1984).

[16] Z. Górny, J. Sobczak, Non-Ferrous Metals Based Novel Materials in Foundry Practice, ZA-PIS, Kraków 2005. 